\title{
Ligante geopolimérico produzido com cinza volante e hidróxido de sódio
}

\author{
Geopolymetric binder produced with fly ash and sodium \\ hydroxide
}

\begin{tabular}{|c|c|}
\hline & $\begin{array}{l}\text { Carolina Noda Livi } \\
\text { Wellington Longuini Repette }\end{array}$ \\
\hline & 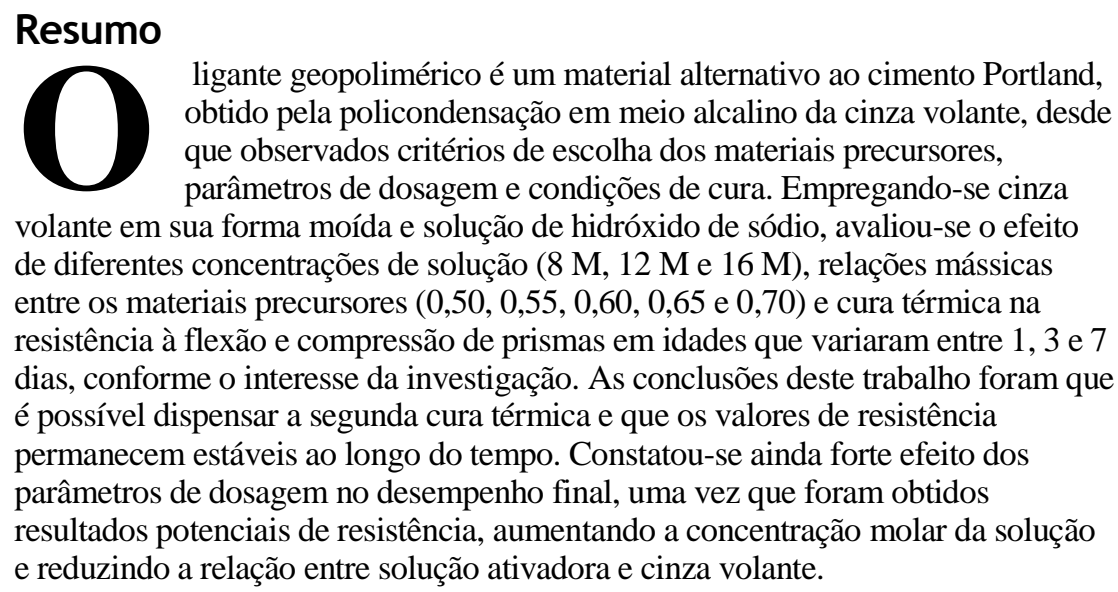 \\
\hline & $\begin{array}{l}\text { Palavras-chaves: Geopolímero. Cinza volante. Solução alcalina. Idade. Resistência. } \\
\text { Abstract }\end{array}$ \\
\hline $\begin{array}{r}\text { Carolina Noda Livi } \\
\text { Universidade Federal de Santa } \\
\text { Catarina } \\
\text { Florianópolis - SC - Brasil }\end{array}$ & $\begin{array}{l}\text { Geopolymeric binders are an alternative material to Portland cement that it are } \\
\text { obtained by fly ash polycondensation in an alkaline medium, as long as criteria for } \\
\text { selection of precursor materials, mixing parameters and curing regime are } \\
\text { observed. By employing ground fly ash and a sodium hydroxide solution, the effect } \\
\text { on flexural and compressive strength of prismatic specimens was evaluated } \\
\text { through changing solution concentration }(8 \mathrm{M}, 12 \mathrm{M} \text { and } 16 \mathrm{M}) \text {, fly ash - alkaline } \\
\text { solution ratio by mass }(0.50,0.55,0.60,0.65,0.70) \text {, curing regime (in oven or }\end{array}$ \\
\hline $\begin{array}{r}\text { Wellington Longuini Repette } \\
\text { Universidade Federal de Santa } \\
\text { Catarina } \\
\text { Florianópolis - SC - Brasil }\end{array}$ & $\begin{array}{l}\text { moist chamber) and age (1, } 3 \text { or } 7 \text { days) according to the aim of each evaluation. } \\
\text { The findings of this study were that a second thermal curing may be dismissed and } \\
\text { strength values remained stable over time. In addition, the investigation showed a } \\
\text { strong effect between mixing parameters, since strength was improved by } \\
\text { increasing the molar concentration and reducing the materials mass ratio. }\end{array}$ \\
\hline $\begin{array}{r}\text { Recebido em 24/08/14 } \\
\text { Aceito em 08/04/15 }\end{array}$ & Keywords: Geopolymer. Fly ash. Alkaline solution. Age. Strength. \\
\hline
\end{tabular}

LIVI, C. N.; REPETTE, W. L. Ligante geopolimérico produzido com cinza volante e hidróxido de sódio. Ambiente 


\section{Introdução}

Geopolímeros são polímeros inorgânicos que possuem uma estrutura amorfa ou semicristalina, dependendo dos materiais empregados e das condições de cura. Embora não possuam uma composição estequiométrica clara, costumam ser representados por uma fórmula empírica, expressa na Equação 1 (DAVIDOVITS, 1991; HARDJITO; RANGAN, 2005).

$\mathrm{M}_{n}\left[-\left(\mathrm{SiO}_{2}\right)_{z}-\mathrm{Al}_{2} \mathrm{O}_{3}\right]_{n} \cdot \mathrm{wH}_{2} \mathrm{O}$

Eq. 1

Onde:

$M$ : metal alcalino ou alcalino-terroso;

$n$ : grau de polimerização;

$z$ : tem valor 1,2 ou 3 , segundo a razão atômica entre silício ( $\mathrm{Si}$ ) e alumínio (Al); e

$w$ : número de moléculas de água.

Da razão atômica entre silício e alumínio, definida no parâmetro $Z$ da Equação 1, surgiu a classificação dos geopolímeros apresentada na Tabela 1. São três grupos: polissialato (M-PS), polissialatossiloxo (M-PSS) e polissialatodissiloxo (M-PSDS). Novamente, $M$ é o metal alcalino na forma de cátion, e $n$ é o grau de polimerização. Já "sialato" é a unidade base e abreviação para silicooxoaluminato $(-S i-O-A l-)$, que consiste em uma rede de tetraedos de $\mathrm{SiO}_{4}$ e $\mathrm{AlO}_{4}$, ligados entre si pelo compartilhamento do oxigênio (DAVIDOVITS, 1991, 1999). A estabilidade do sistema é garantida pelos cátions alcalinos, que atuam como balanceadores de carga das cadeias (DAVIDOVITS, 1991; PALOMO, GRUTZECK; BLANCO, 1999).

É válido mencionar que o termo "geopolímero" foi consagrado pelo pesquisador francês Joseph Davidovits em 1976 (DAVIDOVITS, 1999) e é relacionado a uma classe ampla de ligantes à base de minerais aluminosilicatos (VAN JAARSVELD, VAN DEVENTER; LORENZEN, 1997; DUXSON et al., 2007). A base da química das reações de "geopolimerização" é o processo de ativação alcalina de materiais inorgânicos (KHALE; CHAUNDHARY, 2007) e compreende etapas de dissolução dos sólidos reagentes e transferência das espécies dissolvidas para uma fase gel, seguida de reorganização, polimerização e enrijecimento (DAVIDOVITS, 1999; XU; VAN DEVENTER, 2003; VAN JAARSVELD, VAN DEVENTER; LORENZEN, 1997; DUXSON et al., 2007).

Diversos fatores podem influenciar as reações, como características dos materiais precursores, além das condições de cura, seja temperatura, duração ou tipo (HARDJITO; RANGAN, 2005); a relação solução ativadora por fonte de aluminossilicato (KOMLJENOVIĆ, BAŠČAREVIĆ; BRADIĆ, 2010) e, indiretamente, a quantidade de água (VAN JAARSVELD, VAN DEVENTER; LUKEY, 2003; HARDJITO; RANGAN, 2005).

Os materiais precursores, ricos em sílica $\left(\mathrm{SiO}_{2}\right)$ e alumina $\left(\mathrm{Al}_{2} \mathrm{O}_{3}\right)$, podem ser de fontes naturais, como, por exemplo, o caulim (DAVIDOVITS, 1991; XU; VAN DEVENTER, 2003; BARBOSA, MACKENZIE; THAUMATURGO, 2000) e pozolanas (BONDAR, LYNDSALE; RAMEZANIANPOUR, 2005); ou resíduos industriais: metacaulim proveniente da indústria de papel (SANTA, 2012), cinza volante (HARDJITO; RANGAN， 2005; PALOMO, GRUTZECK; BLANCO, 1999; DIAZ-LOYA, ALLOUCHE; VAIDYA, 2011), cinza pesada (BLISSARI et al., 2011; CHINDAPRASIRT, et al., 2009) e escória de alto-forno combinada com cinza volante (SABITHA et al., 2012; ISMAIL et al., 2014).

Destes os mais utilizados são o metacaulim, a cinza volante e a escória de alto forno (REPETTE, 2010), com destaque para a cinza volante em virtude da disponibilidade em diversas regiões do mundo, devido ao uso crescente de carvão como combustível em usinas termelétricas (AMERICAN..., 2012). Além disso, o emprego de resíduos industriais é uma solução ambientalmente interessante, tendo em vista as preocupações crescentes relacionadas aos impactos ambientais do aquecimento global decorrente dos gases de efeito estufa liberados em diversos setores da indústria.

Tabela 1 - Classificação dos geopolímeros

\begin{tabular}{l|c|c|l}
\hline CLASSE & Si:Al & UNIDADE REPETIDORA & \multicolumn{1}{c}{ APLICAÇÃO } \\
\hline M-PS & $1: 1$ & $M_{n}-(S i-O-A l-O)_{n}$ & $\begin{array}{l}\text { Blocos, materiais cerâmicos, proteção } \\
\text { ao fogo }\end{array}$ \\
\hline M-PSS & $2: 1$ & $M_{n}-(S i-O-A l-O-S i-O)_{n}$ & $\begin{array}{l}\text { Cimentos com baixo teor de } \mathrm{CO}_{2}, \\
\text { encapsulamento de resíduos }\end{array}$ \\
\hline M-PSDS & $3: 1$ & $M_{n}-(S i-O-A l-O-S i-O-S i-O)_{n}$ & $\begin{array}{l}\text { Compósitos com alta resistência ao } \\
\text { calor }\end{array}$ \\
\hline
\end{tabular}

Fonte: adaptado de Davidovits (1999).

8 Livi, C. N.; Repette, W. L. 
A cinza volante é definida por uma combinação de fases vítreas e amorfas, constituída principalmente pelos óxidos de sílica $\left(\mathrm{SiO}_{2}\right)$, alumina $\left(\mathrm{Al}_{2} \mathrm{O}_{3}\right)$, hematita $\left(\mathrm{Fe}_{2} \mathrm{O}_{3}\right)$ e cal $(\mathrm{CaO})$, sendo os três primeiros os óxidos ácidos que reagem com os álcalis do ativador (XIE; XI, 2001). Destes a sílica é o principal constituinte e responsável pela resistência mecânica (FERNÁNDEZ-JIMÉNEZ; PALOMO, 2003).

A composição química da cinza varia tanto para lotes de mesma matéria-prima quanto para fontes diferentes dispersas pelo mundo, em função da origem do carvão mineral, dos procedimentos de coleta, dos métodos de combustão e do próprio formato das partículas (HARDJITO; RANGAN, 2005), o que torna complexo o estudo da influência dos óxidos no comportamento mecânico do geopolímero. Contudo, em um trabalho experimental de avaliação do potencial de reatividade, autores concluíram que o teor ideal de carbono na cinza é inferior a 5\%, de hematita é, no máximo, $10 \%$, enquanto a sílica reativa deve estar presente em $40 \%$ a $50 \%$ (FERNÁNDEZJIMÉNEZ; PALOMO, 2003).

Conforme já definido, a geopolimerização se dá em meio alcalino. A solução ativadora dissolve o silício e alumínio por hidrólise da superfície das partículas (VAN JAARSVELD, VAN DEVENTER; LORENZEN, 1997; DUXSON et al., 2007; KHALE; CHAUNDHARY, 2007), além de atuar como catalisador das reações (XU; VAN DEVENTER, 2000). Usualmente, as soluções ativadoras são compostas de silicatos solúveis e hidróxidos alcalinos (HARDJITO; RANGAN, 2005), todavia algumas pesquisas são conduzidas apenas com hidróxido de sódio em sua composição (SOMMA et al., 2011; VARGAS et al., 2011; CRIADO, FERNÁNDEZ-JIMÉNEZ; PALOMO, 2010).

Por fim, uma solução ativadora com elevada concentração molar resulta em geopolímeros com forte aderência da fase gel (XU; VAN DEVENTER, 2003) e melhor resistência mecânica (FERNÁNDEZ-JIMÉNEZ; PALOMO, 2005), até uma dosagem ótima a partir da qual o excesso provoca perda de resistência (PALOMO; GRUTZECK; BLANCO, 1999).

Em função dos materiais precursores adotados, deve-se selecionar o regime de cura mais adequado. No caso de ligantes geopoliméricos à base de cinza volante e hidróxido de sódio, objeto de estudo deste artigo, adota-se a cura térmica, normalmente realizada em estufa (HARDJITO; RANGAN, 2005; PALOMO; GRUTZECK; BLANCO, 1999; DIAZ-LOYA, ALLOUCHE; VAIDYA, 2011), podendo ocorrer em banho térmico (CRIADO et al., 2007; OH et al., 2010), câmara climatizada, precedida ou não por uma cura inicial em sala climatizada (HARDJITO; RANGAN, 2005), ou forno micro-ondas (CHINDAPRASIRT, RATTANASAK; TAEBUANHUAD, 2013). A partir de uma granulometria adequada e de um processo de mistura diferenciado, pode-se dispensar a cura térmica (SOMMA et al., 2011).

Da escolha correta dos materiais precursores, dosagem e condições de cura, os geopolímeros produzidos apresentam alta resistência à compressão (VAN JAARSVELD; VAN DEVENTER; LORENZEN, 1997; DUXSON et al., 2007; XU; VAN DEVENTER, 2003; PALOMO; ALONSO; FERNÁDEZ-JIMÉNEZ, 2004), principalmente nas primeiras idades (HARDJITO; RANGAN, 2005). É um material que apresenta boa resistência ao ataque de ácidos (VAN JAARSVELD; VAN DEVENTER; LORENZEN, 1997; DUXSON et al., 2007; XU; VAN DEVENTER, 2003; DAVIDOVITS, 1994) e à ação do fogo (DUXSON et al., 2007; XU; VAN DEVENTER, 2003). No que tange às propriedades do estado fresco, a pasta geopolimérica possui viscosidade elevada o suficiente para prevenir a segregação das partículas, contudo sem inviabilizar a moldagem (VAN JAARSVELD; VAN DEVENTER; LORENZEN, 1997), sendo possível ajustar o tempo de pega conforme a necessidade (VAN JAARSVELD; VAN DEVENTER; LORENZEN, 1997; DUXSON et al., 2007).

Além da aplicação como ligante alternativo na produção de argamassas e concretos, os geopolímeros foram introduzidos na indústria de materiais de construção com sucesso (DAVIDOVITS, 1991; XU; VAN DEVENTER, 2000). São empregados, por exemplo, na imobilização de metais e resíduos tóxicos através da incorporação destes à matriz geopolimérica, na indústria de pré-moldados e protendidos, além de etapas de recapeamento em pista de aeroporto ou estradas (DAVIDOVITS, 1991; VAN JAARSVELD; VAN DEVENTER; LORENZEN, 1997). Diferentes composições estão sendo investigadas para aplicação na cimentação de poços de petróleo (PINTO, 2011; SANTOS, 2012).

Portanto, através da geopolimerização são desenvolvidas características parecidas às do cimento Portland, com o diferencial de apresentar benefícios econômicos e ambientais, decorrentes do emprego de resíduos industriais e confecção de cimento alternativo. As fontes de produção do clínquer são preservadas, e há menor consumo de energia e emissão de dióxido de carbono $\left(\mathrm{CO}_{2}\right)$ (FERNÁNDEZ-JIMÉNEZ; PALOMO, 2005), já 
que sua obtenção independe da calcinação do calcário e argila para produzir o pó ligante.

Neste contexto, objetivou-se avaliar o efeito na resistência à flexão e compressão de prismas, de diferentes composições de pastas de cinza volante ativadas com hidróxido de sódio, condições de moldagem, valores de dosagem e condições de cura.

\section{Materiais e programa experimental}

\section{Materiais precursores}

A fonte de aluminossilicato é a cinza volante proveniente da Central Termoelétrica Jorge Lacerda (Capivari de Baixo, SC), da Tractebel Energia, cuja composição química segundo análise de fluorescência de raios $X$ é apresentada na Tabela 2. A massa específica, determinada pelo método do picnômetro, é de $2,49 \mathrm{~g} / \mathrm{cm}^{3}$.
A cinza foi moída por cinco ciclos de $110 \mathrm{~min}$ em moinho de bolas AMEF, modelo AMBI 480 x 400, com 120 bolas de aço de $29,7 \mathrm{~mm}$ de diâmetro e $1.310 \mathrm{~g}$ de massa unitária, em conjunto com 150 bolas de $19,2 \mathrm{~mm}$ e $360 \mathrm{~g}$. Com esse procedimento, obteve-se um material tão fino quanto um cimento $\mathrm{CP}$ V-ARI, com diâmetro médio (D50) igual a $16 \mu \mathrm{m}$ (LIVI, 2013). A Figura 1 permite comparar as diferentes distribuições granulométricas, obtidas por granulometria a laser, da cinza volante original, moída, e de uma amostra de cimento Portland CP V-ARI.

A solução ativadora responsável pela ativação conteve hidróxido de sódio PA em micropérolas da Vetec nas pastas identificadas "A" a "E" e hidróxido de sódio em escamas da carbocloro nas demais, ambas com teor de alcalinidade total superior a $98 \%$.

Tabela 2 - Composição química da cinza volante

\begin{tabular}{c|c}
\hline ELEMENTO & CINZA VOLANTE \\
\hline $\mathrm{SiO}_{2}$ & 64,1 \\
\hline $\mathrm{Al}_{2} \mathrm{O}_{3}$ & 22,9 \\
\hline $\mathrm{Fe}_{2} \mathrm{O}_{3}$ & 2,2 \\
\hline $\mathrm{K}_{2} \mathrm{O}$ & 2,0 \\
\hline $\mathrm{CO}_{2} *$ & 1,5 \\
\hline $\mathrm{CaO}$ & 1,4 \\
\hline $\mathrm{TiO} \mathrm{O}_{2}$ & 1,4 \\
\hline $\mathrm{MgO}$ & 0,6 \\
\hline $\mathrm{Na}_{2} \mathrm{O}$ & 0,2 \\
\hline $\mathrm{SO}_{3}$ & 0,2 \\
\hline $\mathrm{P}_{2} \mathrm{O}_{5}$ & 0,10 \\
\hline $\mathrm{MnO}$ & 0,00 \\
\hline
\end{tabular}

Figura 1 - Distribuição granulométrica

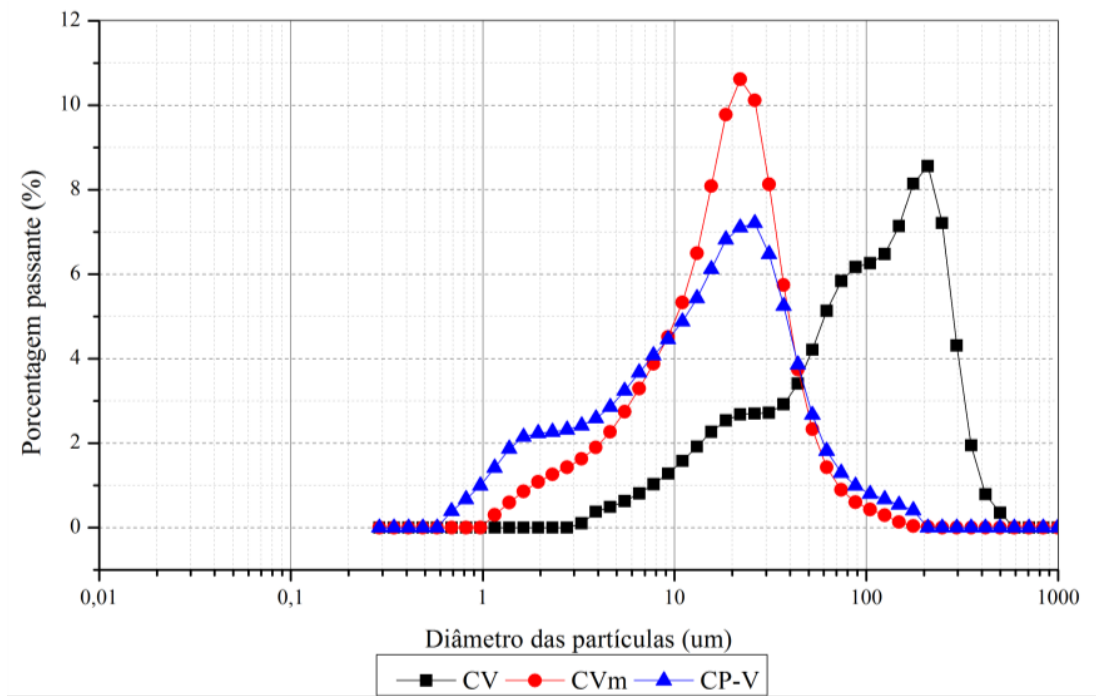

Fonte: Livi (2013).

10 Livi, C. N.; Repette, W. L. 


\section{Proporcionamento das misturas}

Foram propostas oito misturas, descritas na Tabela 3, para avaliar o efeito de uma segunda cura térmica, parâmetros de dosagem (relação de massa entre a solução ativadora e a cinza volante; concentração molar da solução ativadora), idade na resistência à flexão e compressão. Quanto ao efeito do agente desmoldante, empregou-se o spray de silicone nas misturas "A" a "D", e "H". Uma vez que em alguns casos parte do material permanecia aderida, para as misturas "E", "F", "G" optou-se pelo envelopamento das paredes do molde com filme plástico de PVC.

Foram preparadas soluções ativadoras com concentração molar de 8,12 e $16 \mathrm{M}$, em balão volumétrico de $500 \mathrm{~mL}$, dissolvendo-se o hidróxido de sódio sólido em água destilada. A quantidade de soluto foi determinada segundo a Equação 2.

$[\mathrm{NaOH}]=\frac{m}{M . V}$

Eq. 2

Onde:

[NaOH]: concentração molar (ou molaridade) da solução de hidróxido de sódio (M ou mol/L);

$m$ : massa de soluto $(\mathrm{g})$;

$M$ : massa molecular do soluto (40 g/mol); e

$V$ : volume do balão volumétrico $(500 \mathrm{~mL})$.

Tabela 3 - Detalhamento das misturas avaliadas

\begin{tabular}{|c|c|c|c|c|}
\hline MISTURA & $\begin{array}{l}\text { EFEITO A SER } \\
\text { AVALIADO }\end{array}$ & $\begin{array}{l}\text { CONCENTRAÇÃO } \\
\text { MOLAR (M) }\end{array}$ & $\begin{array}{c}\text { SOLUÇÃO } \\
\text { ATIVADORA/CINZA } \\
\text { VOLANTE }(\mathbf{g} / \mathrm{g})\end{array}$ & $\begin{array}{l}\text { IDADES } \\
\text { (dias) }\end{array}$ \\
\hline A & $\begin{array}{l}\text { Segunda cura } \\
\text { térmica, relação } \\
\text { solução ativadora } \\
\text { por cinza volante }\end{array}$ & 16 & 0,50 & 3 \\
\hline B & $\begin{array}{l}\text { Segunda cura } \\
\text { térmica, relação } \\
\text { solução ativadora } \\
\text { por cinza volante }\end{array}$ & 16 & 0,60 & 3 \\
\hline $\mathrm{C}$ & $\begin{array}{l}\text { Segunda cura } \\
\text { térmica, relação } \\
\text { solução ativadora } \\
\text { por cinza volante }\end{array}$ & 16 & 0,70 & 3 \\
\hline $\mathrm{D}$ & $\begin{array}{l}\text { Idade e concentração } \\
\text { molar }\end{array}$ & 8 & 0,55 & $1,3,7$ \\
\hline $\mathrm{E}$ & $\begin{array}{l}\text { Relação solução } \\
\text { ativadora por cinza } \\
\text { volante, desmoldante }\end{array}$ & 12 & 0,50 & 3 \\
\hline $\mathrm{F}$ & $\begin{array}{l}\text { Relação solução } \\
\text { ativadora por cinza } \\
\text { volante, idade, } \\
\text { concentração molar, } \\
\text { desmoldante }\end{array}$ & 12 & 0,55 & $1,3,7$ \\
\hline G & $\begin{array}{l}\text { Relação solução } \\
\text { ativadora por cinza } \\
\text { volante, desmoldante }\end{array}$ & 12 & 0,60 & 3 \\
\hline $\mathrm{H}$ & $\begin{array}{l}\text { Relação solução } \\
\text { ativadora por cinza } \\
\text { volante, idade e } \\
\text { concentração molar }\end{array}$ & 12 & 0,65 & $1,3,7$ \\
\hline
\end{tabular}

Nota: Legenda:

ID - identificação;

M - concentração molar; e

$\mathrm{S}$ - relação solução ativadora por cinza volante, em massa. 
O proporcionamento das pastas foi dado em volume, de modo que foi necessário determinar a massa específica da solução ativadora $\left(\mathrm{d}_{\mathrm{SA}}\right.$, em $\mathrm{g} / \mathrm{cm}^{3}$ ), dividindo-se a massa por seu volume. Adicionalmente, a título de caracterização, foi calculado o teor sólido das soluções (Equação 3), segundo metodologia proposta por Hardjito e Rangan (HARDJITO; RANGAN, 2005).

$\tau=\frac{[N a O H] \cdot \mathrm{M}}{d_{S A}}$

Eq. 3

Onde $\tau$ teor de sólidos, isto é, a massa do soluto em relação à solução (expresso em valor absoluto).

Para a concentração de $8 \mathrm{M}$, os valores médios de massa específica e teor de sólidos foram respectivamente $1,24 \mathrm{~g} / \mathrm{cm}^{3}$ e 0,26 , enquanto para $12 \mathrm{M}$ foram $1,34 \mathrm{~g} / \mathrm{cm}^{3}$ e 0,36 . Por fim, uma molaridade igual a $16 \mathrm{M}$ produziu um líquido alcalino com massa específica média de $1,42 \mathrm{~g} / \mathrm{cm}^{3}$ e teor de sólidos de 0,45 .

\section{Procedimento: amassamento, moldagem, cura e caracterização dos corpos de prova}

As pastas foram preparadas em agitador mecânico Tedemix com frequência de agitação de $25 \mathrm{~Hz}$, equivalente a 733 RPM, durante 5 min. Foram moldados três corpos de prova prismáticos, nas dimensões de $4 \mathrm{~cm}$ x $4 \mathrm{~cm}$ x $16 \mathrm{~cm}$ para cada composição.

A cura térmica ocorreu em estufa microprocessada com circulação forçada de ar Quimis Q314M, durante $22 \mathrm{~h}$, na temperatura de $85^{\circ} \mathrm{C}$.

Após 24 h, os corpos de prova foram desmoldados. Realizou-se a medição das dimensões e da massa de cada exemplar, em quatro repetições, para calcular a massa específica de cada corpo de prova endurecido, dada pela relação entre sua massa e volume. Variou de $1,7 \mathrm{~g} / \mathrm{cm}^{3}$ até $1,8 \mathrm{~g} / \mathrm{cm}^{3}$, sem diferenças significativas, para inferir se há relação com a concentração da solução ou relação mássica entre os materiais.

Por fim, os corpos de prova foram acondicionados segundo as variantes investigadas. Salvo exceção, que será mencionada no item a seguir - Resultados e discussões, os demais corpos de prova foram mantidos em câmara úmida até a data dos ensaios mecânicos.

\section{Avaliação das propriedades mecânicas}

Os corpos de prova foram submetidos aos ensaios de resistência à flexão e compressão, com base na norma BS EN 196-1:2005 -Methods of testing cement - Part 1: Determination of strength. As idades de ruptura variaram entre 1, 3 e 7 dias, conforme o interesse da investigação.

O ensaio de flexão foi conduzido a uma taxa de carregamento de $(50 \pm 10) \mathrm{N} / \mathrm{s}$, em prensa computadorizada Instron 5569. A resistência de ruptura foi calculada pela Equação 4 .

$R_{f}=\frac{1,5 \cdot F_{f} \cdot l}{b \cdot h^{2}}$

Eq. 4

Onde:

$R_{f}$ : valor de resistência à flexão $(\mathrm{MPa})$;

$F_{f}$ : carga de ruptura de flexão $(\mathrm{N})$;

$l$ : distância entre os apoios (mm);

$b$ : lado da seção (mm); e

$h$ : altura da seção (mm).

Cada metade de prisma, obtida do procedimento anterior, foi ensaiada à compressão com uma taxa de aplicação de carga de $(2.400 \pm 200)$ N/s em prensa de ensaio universal Shimadzu e tensão calculada a partir da Equação 5.

$R_{c}=\frac{F_{C}}{1600}$

Eq. 5

Onde:

$R_{c}$ : valor de resistência à compressão $(\mathrm{MPa})$;

$F_{c}$ : carga de ruptura de compressão $(\mathrm{N})$; e

1600: área de aplicação de carga (40 mm x 40 $\mathrm{mm})$.

\section{Resultados e discussões}

\section{Quanto à segunda cura térmica}

Silverstrim et al. (1997), em sua patente destinada à obtenção de materiais cimentícios a partir da ativação química da cinza volante, sugerem uma segunda cura térmica em menor temperatura após o desmolde dos corpos de prova. Verificou-se se havia necessidade desse procedimento, isto é, se o ganho de resistência justificava o maior consumo de energia devido à cura prolongada, de modo que os prismas submetidos à segunda cura permaneceram em estufa a $45^{\circ} \mathrm{C}$ durante $24 \mathrm{~h}$ e, posteriormente, foram armazenados na câmara até completar a idade de ensaio de 3 dias. Os resultados de resistência à flexão e compressão podem ser conferidos nas Figuras 2 e 3. 
Figura 2 - Efeito da segunda cura na resistência à flexão

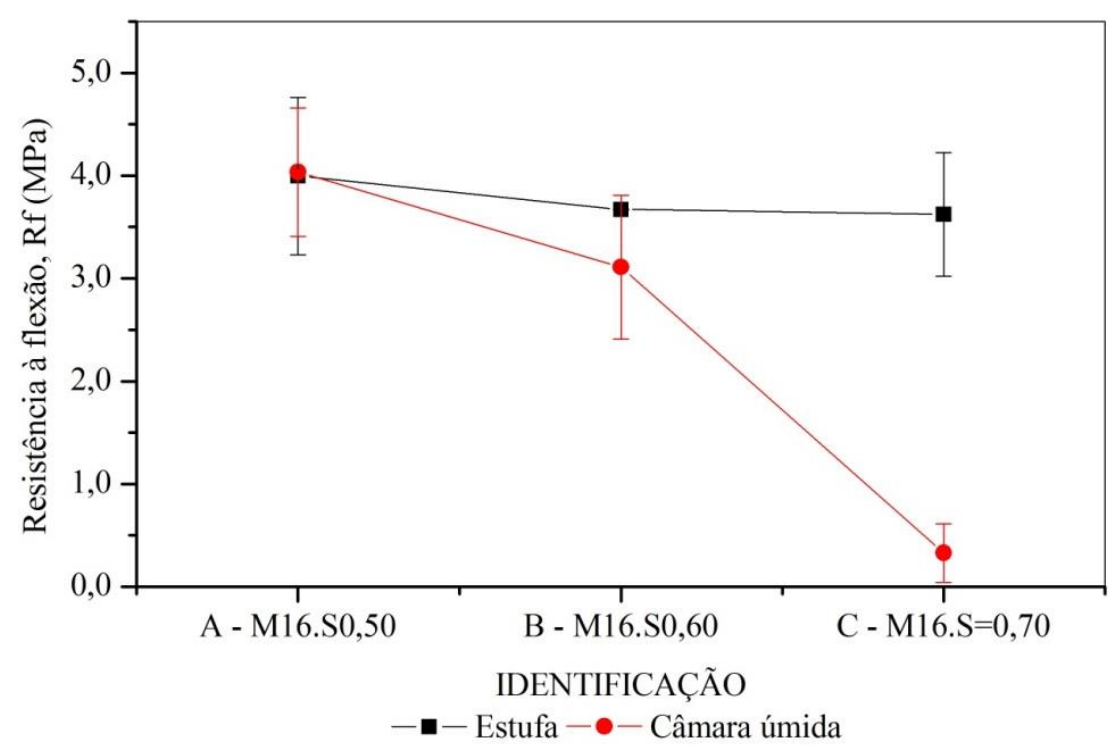

Figura 3 - Efeito da segunda cura na resistência à compressão

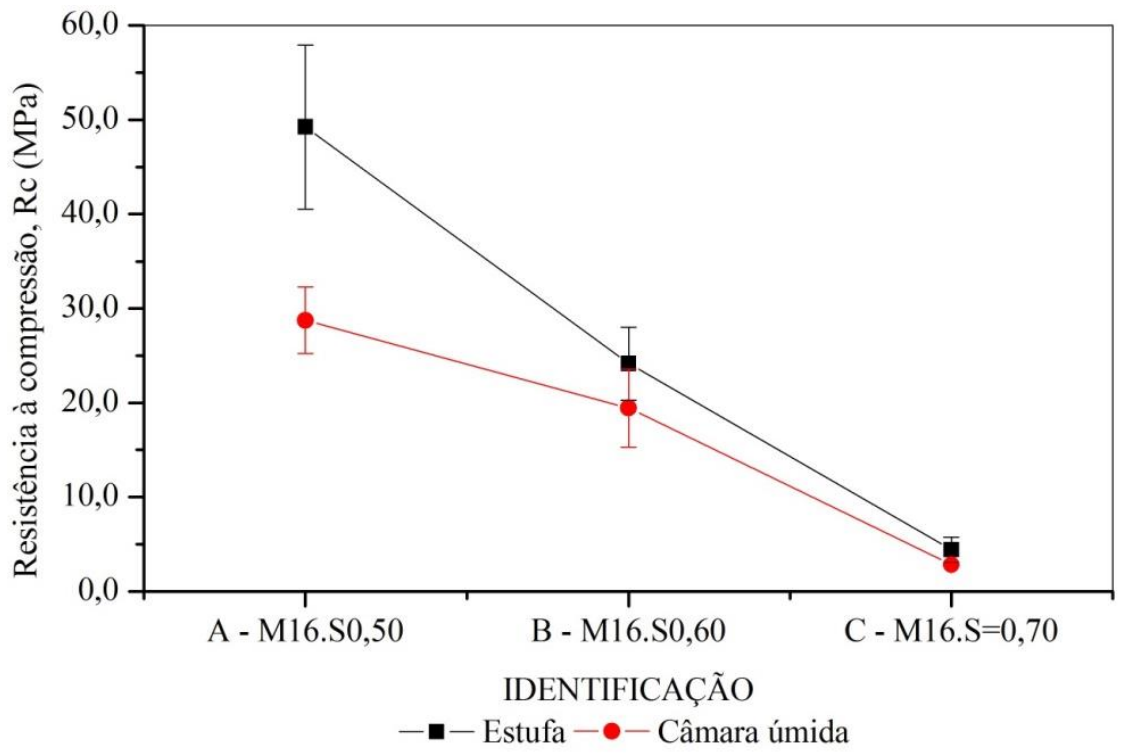

A segunda cura térmica favoreceu o desenvolvimento de resistência, porém, como se observou nos resultados de flexão das pastas " $\mathrm{A}$ " e "B" e de compressão de "B" e "C", as diferenças não foram expressivas. Além disso, não houve tendência de comportamento, pois, enquanto no ensaio de flexão (Figura 2) a pasta "C" apresentou incremento de resistência de 3,29 $\mathrm{MPa}$, na compressão (Figura 3) não foram observadas diferenças expressivas. Da mesma forma, a segunda cura térmica da pasta "A" promoveu melhor resultado na compressão, mas não na flexão. Pelos motivos citados, concluiu-se descartar esse procedimento.

\section{Quanto ao desenvolvimento de resistência ao longo do tempo}

As pastas " $D$ ", " $F$ " e "H" foram rompidas com 1, 3 e 7 dias, e os resultados de resistência à flexão e compressão estão nas Figuras 4 e 5. 
Figura 4 - Monitoramento da resistência à flexão ao longo do tempo

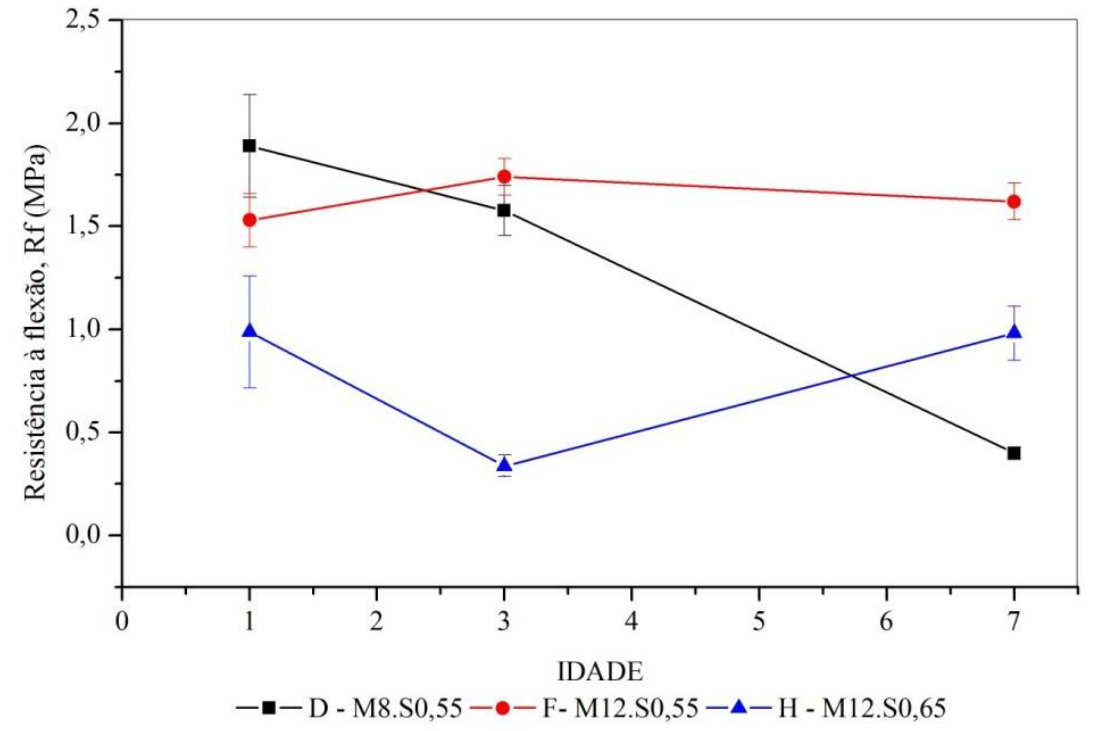

Figura 5 - Monitoramento da resistência à compressão ao longo do tempo

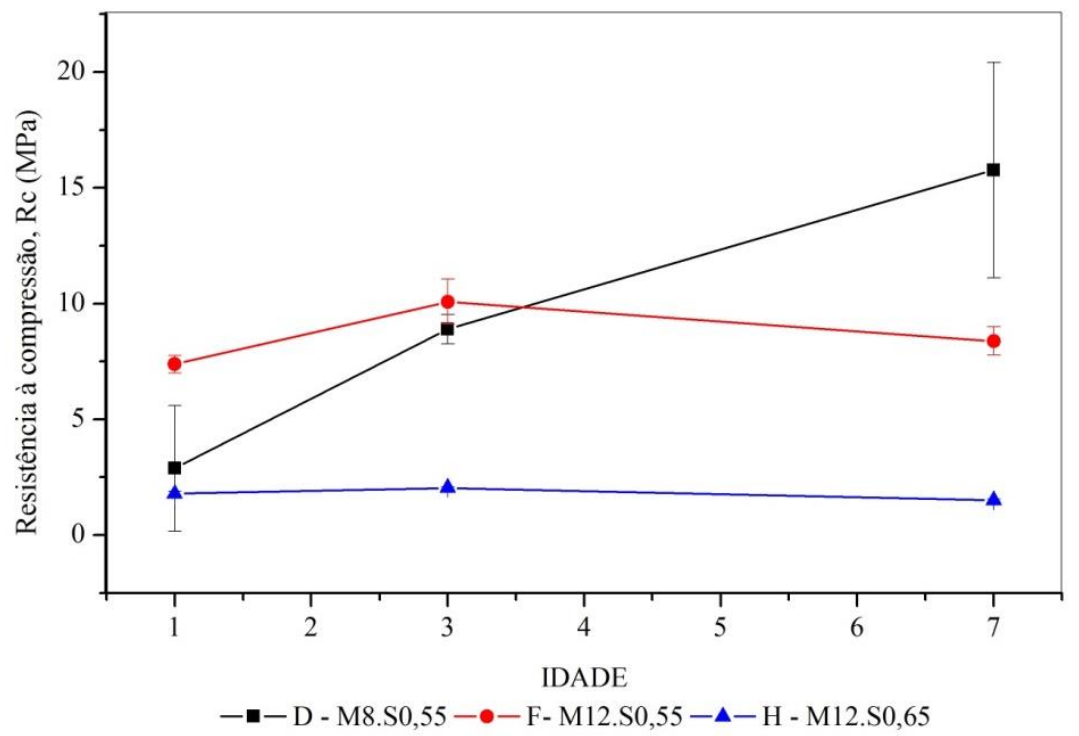

As pastas "D" e "F" apresentaram valores próximos de resistência à flexão a 1 e 3 dias. Aos 7 dias a mistura "D" apresentou menor resistência à flexão e maior à compressão em relação à " $F$ ". Os ganhos de resistência ao longo do tempo foram mínimos e coerentes com dados de literatura, em que autores verificaram a baixa influência da idade no desenvolvimento de resistência dos geopolímeros (HARDJITO; RANGAN, 2005; YOST et al., 2013).

Tais resultados indicam o potencial do ligante geopolimérico para ser utilizado em situações nas quais é necessário rápido desenvolvimento de resistência, para que seja possível pôr em uso o material ainda em baixas idades, como é o caso da indústria de pré-fabricados. Tal potencial foi igualmente mencionado por Van Jaarsveld, Van Deventer e Lorezen (1997).

\section{Quanto à relação solução ativadora por cinza volante e concentração molar da solução alcalina}

O efeito da relação em massa de solução ativadora por cinza volante para uma mesma concentração molar foi investigado para as pastas identificadas com concentração molar igual a 12 e relações de $0,50,0,55,0,60$ e 0,65 . Para uma molaridade igual a 16 estabeleceram-se as relações $0,50,0,60$ e 0,70. Assim, foi possível uma avaliação otimizada dos dois parâmetros de dosagem usualmente avaliados em geopolímeros. Os resultados de 
resistência à flexão de prismas estão na Figura 6, enquanto os de resistência à compressão estão na Figura 7.

Ambos os parâmetros influenciaram os valores de resistência à flexão e compressão, que foram crescentes à medida que se diminuiu a quantidade de líquido alcalino presente na mistura, expresso pela relação solução ativadora por cinza volante, e também pelo aumento da concentração molar da solução alcalina.

Assim como ocorre com o fator água/cimento em misturas cimentícias tradicionais, quanto menor a relação mássica entre solução ativadora e cinza volante, maiores são os resultados de resistência. Porém, diferentemente daquelas, em que a água participa das reações de hidratação e consequente formação de produtos hidratados responsáveis pelo desempenho do material endurecido, em geopolímeros a água é apenas o meio para as reações de dissolução, sendo expulsa com o decorrer da policondensação. Sendo assim, não há uma relação direta entre a quantidade de água e a qualidade dos produtos obtidos, como ocorre nas matrizes de cimento Portland. Todavia, afeta as propriedades da mistura tanto no estado fresco, influenciando a trabalhabilidade, quanto endurecido, no que tange à resistência mecânica, possivelmente por estar associada à porosidade da matriz (HARDJITO; RANGAN, 2005; KOMLJENOVIĆ, BAŠČAREVIĆ; BRADIĆ, 2010).

Figura 6 - Efeito da relação solução ativadora por cinza volante e da concentração molar na resistência à flexão

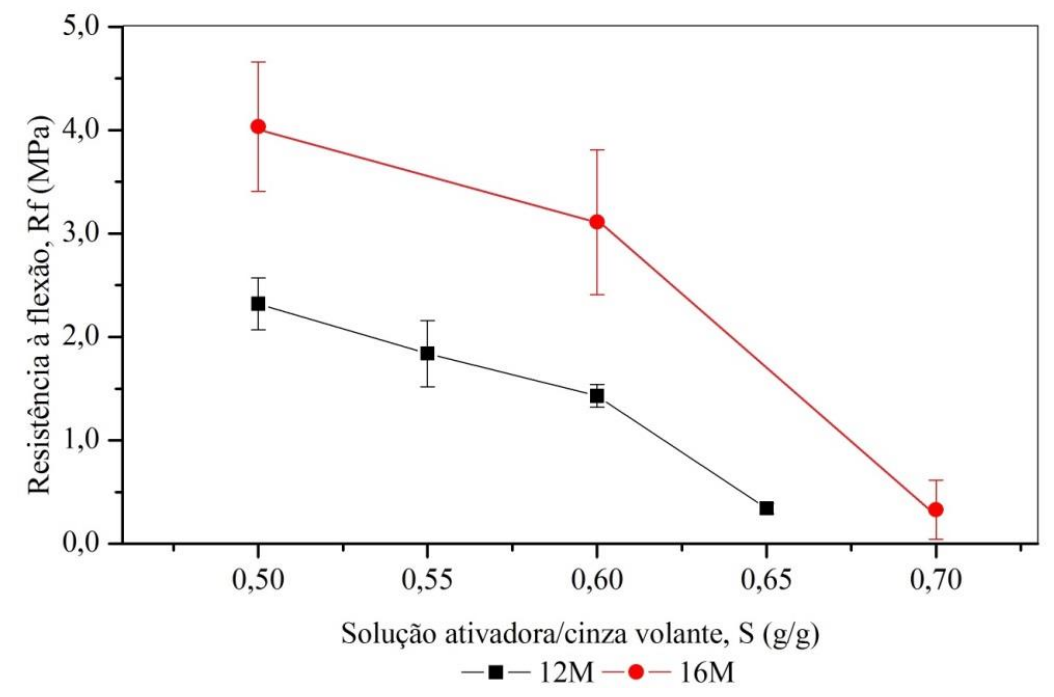

Figura 7 - Efeito da relação solução ativadora por cinza volante e da concentração molar na resistência à compressão

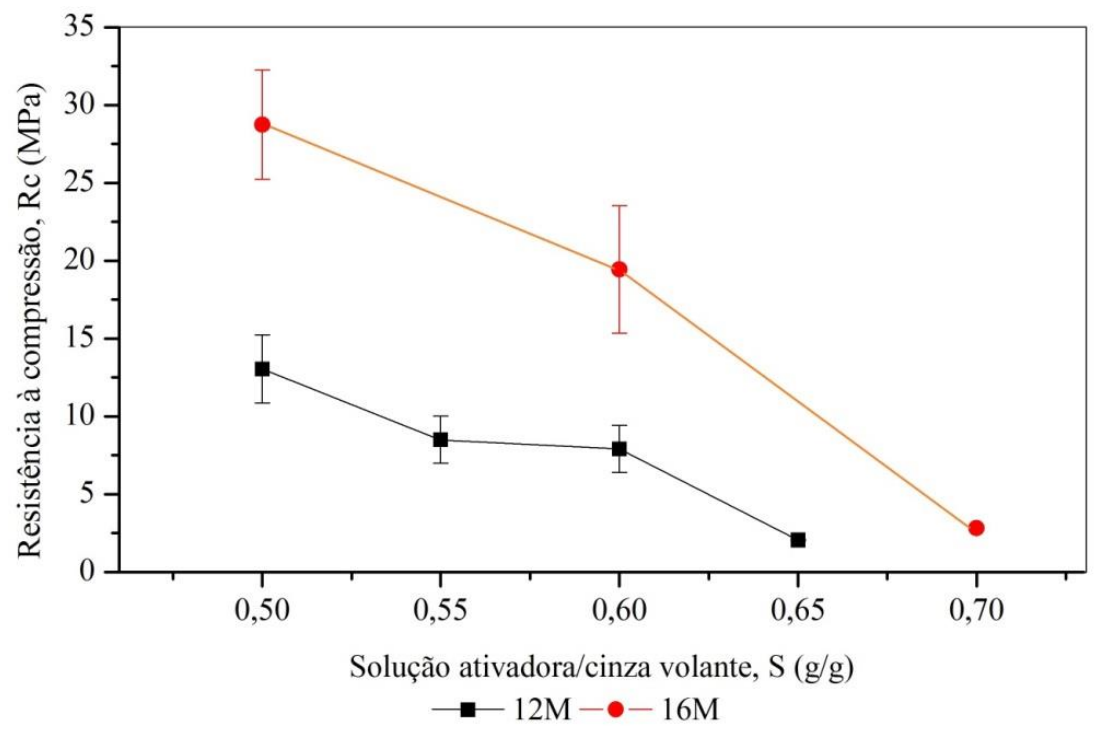


No que diz respeito à concentração molar, para uma mesma relação mássica entre solução ativadora e cinza volante, para uma maior quantidade de íons hidroxila, maior a resistência observada (PALOMO; ALONSO; FERNÁDEZJIMÉNEZ, 2004), porém não foi observado um ponto ótimo de dosagem como fora proposto em Palomo, Grutzcek e Blanco (1999).

\section{Conclusões}

Os resultados deste programa experimental permitiram estabelecer as conclusões a seguir.

No que se refere à técnica de desmoldagem, o uso de filme plástico de PVC envelopando as paredes do molde foi a alternativa mais eficiente para desmoldar o corpo de prova, sem prejudicar sua integridade, com a vantagem de melhorar o acabamento dele.

Quanto ao tipo de cura, concluiu-se ser possível descartar a segunda cura térmica, sem prejuízo nos valores de resistência. Em relação à idade, não foi possível evidenciar a evolução de resistência ao longo dos 7 dias, como já havia sido observado por outros autores (HARDJITO; RANGAN, 2005; YOST et al., 2013)

Por fim, melhorou-se o desempenho mecânico com o aumento da concentração molar ou da diminuição da quantidade de solução ativadora por cinza volante, fato este que está de acordo com constatações de Fernández-Jiménez e Palomo (2005), Hardjito e Rangan (2005), Duxson et al. (2007), Chindaprasirt et al. (2009) e Komljenović, Baščarević e Bradić (2010).

\section{Referências}

\section{AMERICAN SOCIETY FOR TESTING \\ MATERIALS. ASTM C618: standard}

specification for coal fly ash and raw or calcined natural pozzolan in concrete. Pennsylvania, 2012.

BARBOSA, V. F. F.; MACKENZIE, K. J. D.; THAUMATURGO, C. Synthesis and Characterisation of Materials Based on Inorganic Polymers of Alumina and Silica: sodium polysialate polymers. International Journal of Inorganic Materials, v. 2, n. 4, p. 309-317, 2000.

BLISSARI, E. S. et al. Geopolímeros Obtidos a Partir de Cinzas de Carvão Mineral. In: SEMINÁRIO DE PESQUISA, EXTENSÃO E INOVAÇÃO DO INSTITUTO FEDERAL DE SANTA CATARINA, 2011. Anais... Criciúma: IFSC, 2011.
BONDAR, D.; LYNDSALE, C. J.;

RAMEZANIANPOUR, A. A. Alkali Activation of Natural Pozzolan for Geopolymer Cement Production. In: International Conference on Concrete \& Development, 2., Tehran, 2005. Proceedings... Tehran, 2005.

CHINDAPRASIRT, P. et al. Comparative Study on the Characteristics of Fly Ash and Bottom Ash Geopolymers. Waste Management, v. 29, n. 2, p. 539-543, 2009.

CHINDAPRASIRT, P.; RATTANASAK, U.; TAEBUANHUAD, S. Resistance to Acid and Sulfate Solutions of Microwave-Assisted High Calcium Fly Ash Geopolymer. Materials and Structures, v. 46, p. 375-381, 2013.

CRIADO, M. et al. An XRD Study of the Effect of the $\mathrm{SiO}_{2} / \mathrm{Na}_{2} \mathrm{O}$ Ratio on the Alkali Activation of Fly Ash. Cement and Concrete Research, v. 37, n. 5, p. 671-679, 2007.

CRIADO, M.; FERNÁNDEZ-JIMÉNEZ, A.; PALOMO, A. Effect of Sodium Sulfate on the Alkali Activation of Fly Ash. Cement and Concrete Composites, v. 32, n. 8, p. 589-594, 2010.

DAVIDOVITS, J. Geopolymers: inorganic polymeric new materials. Journal of Thermal Analysis, v. 37, n. 8, p. 1633-1656, 1991.

DAVIDOVITS, J.; Geopolymers: man-made rock geosynthesis and the resulting development of very early high strength cement. Journal of Materials Education, v. 16, n. 2/3, p. 91-139, 1994.

DAVIDOVITS, J. Chemistry of Geopolymeric Systems, Terminology. In: Conference Géopolymère, 99., Saint-Quentin, 1999. Proceedings... Saint-Quentin: Institut Géopolymère, 1999.

DIAZ-LOYA, E. I.; ALLOUCHE, E. N.; VAIDYA, S. Mechanical Properties of Fly-AshBased Geopolymer Concrete. ACI Materials Journal, v. 108, n. 3, p. 300-306, may/jun. 2011.

DUXSON, P. et al. Geopolymer Technology: the current state of the art. Journal of Materials Science, v. 42, n. 9, p. 2917-2933, 2007.

FERNÁNDEZ-JIMÉNEZ, A.; PALOMO, A. Characterisation of Fly Ashes: potential reactivity as alkaline cements. Fuel, v. 82, n. 18, p. 22592265, 2003.

FERNÁNDEZ-JIMÉNEZ, A.; PALOMO, A. Composition and Microstructure of Alkali Activated Fly Ash Binder: effect of the activator. Cement and Concrete Research, v. 35, n. 5, p. 1984-1992, 2005. 
HARDJITO, B.; RANGAN, B. V. Development and Properties of Low-Calcium Fly Ash-Based Geopolymer Concrete. Research Report GC1. Faculty of Engineering, Curtin University of Technology. Perth, Australia, 2005.

ISMAIL, I. et al. Modification of Phase Evolution in Alkali-Activated Blast Furnace Slag by the Incorporation of Fly Ash. Cement \& Concrete Composites, v. 45, p. 125-135, 2014.

KHALE, D.; CHAUNDHARY, R. Mechanism of Geopolymerization and Factors Influencing Its Development: a review. Journal of Materials Science, v. 42, n. 3, p. 729-746, 2007.

KOMLJENOVIĆ, M.; BAŠČAREVIĆ, Z.; BRADIĆ, V. Mechanical and Microstructural Properties of Alkali-Activated Fly Ash Geopolymers. Journal of Hazardous Materials, v. 181, n. 1/3, p. 35-42, 2010.

LIVI, C. N. Desenvolvimento de Pasta de Geopolímero a Base de Cinza Volante e Hidróxido de Sódio. Florianópolis, 2013. 193 f. Dissertação (Mestrado em Engenharia Civil) Programa de Pós-Graduação em de Engenharia Civil, Universidade Federal de Santa Catarina, Florianópolis, 2013.

$\mathrm{OH}, \mathrm{J}$. E. et al. The Evolution of Strength and Crystalline Phases For alkali-Activated Ground Blast Furnace Slag and Fly Ash-Based Geopolymers. Cement and Concrete Research, v. 40, n. 2, p. 189-196, 2010.

PALOMO, A.; ALONSO, S.; FERNÁDEZJIMÉNEZ, A. Alkaline Activation of Fly Ashes: NMR Study of the reaction products. Journal of the American Ceramic Society, v. 87, n. 6, p. 1141-1145, 2004.

PALOMO, A.; GRUTZECK, M. W.; BLANCO, M. T. Alkali-Activated Fly Ashes: a cement for the future. Cement and Concrete Research, v. 29, n. 8, p. 1323-1329, 1999.

PINTO, N. D. M. G. Adição de Resíduo Cerâmico em Pastas Geopoliméricas Para Cimentação de Poços de Petróleo. Natal, 2011. 94 f. Tese (Doutorado em Engenharia Civil) Programa de Pós-Graduação em Ciência e Engenharia de Materiais, Centro de Ciências Exatas e da Terra, Universidade Federal do Rio Grande do Norte, Natal, 2011.

REPETTE, W. L. Cimentos Especiais de Base Mineral. In: ISAIA, G. C. Materiais de Construção Civil e Princípios de Ciência e Engenharia de Materiais. 2. ed. São Paulo: IBRACON, 2010.
SABITHA, D. et al. Reactivity, Workability and Strength of Potassium Versus Sodium-Activated Hhigh Volume Fly Ash-Based Geopolymers. Current Science, v. 103, n. 11, p. 1320-1327, 2012.

SANTA, R. A. A. B. Desenvolvimento de Geopolímeros a Partir de Cinzas Pesadas Oriundas da Queima do Carvão Mineral e Metacaulim Sintetizado a Partir de Resíduo da Industria de Papel. Florianópolis, 2012. 134 f. Dissertação (Mestrado em Engenharia Civil) Programa de Pós-Graduação em Engenharia Química, Universidade Federal de Santa Catarina, Florianópolis, 2012.

SANTOS, E. A. D. Influência da adição de diatomita em pastas geopoliméricas para poços de petróleo. Natal. 2012.

SILVERSTRIM, T. et al. Fly Ash Cementitious Material and Method of Making a Product. US Patent 5.601.643, 11 Fevereiro 1997.

SOMMA, K. et al. NaOH-Activated Ground Fly Ash Geopolymer Cured at Ambient Temperature. Fuel, v. 90, p. 2118-2124, 2011.

VAN JAARSVELD, J. G. S.; VAN DEVENTER, J. S. J.; LORENZEN, L. The Potential Use of Geopolymeric Materials to Immobilise Toxic Metals: part I: theory and applications. Minerals Engineering, v. 10, n. 7, p. 659-669, 1997.

VAN JAARSVELD, J. G. S.; VAN DEVENTER, J. S. J.; LUKEY, G. C. The Characterization of Source Materials in Fly Ash-Based Geopolymers. Materials Letters, v. 57, n. 7, p. 1272-1280, 2003.

VARGAS, A. S. D. et al. The Effects of $\mathrm{Na}_{2} \mathrm{O} / \mathrm{SiO}_{2}$ Molar Ratio Curing Temperature and Age on Compressive Strength, Morphology and Microstructure of Alkali-Activated Fly Ash-Based Geopolymers. Cement and Concrete Composites., v. 33, p. 653-660, 2011.

XIE, Z.; XI, Y. Hardening Mechanisms of an Alkaline-Activated Class F Fly Ash. Cement and Concrete Research, v. 31, n. 9, p. 1245-1249, 2001.

XU, H.; VAN DEVENTER, J. S. J. The Geopolymerisation of Alumino-Silicate Minerals. International Journal of Mineral Processing, v. 59, n. 3, p. 247-266, 2000.

XU, H.; VAN DEVENTER, J. S. J. The Effect of the Alkalis Metals on the Formation of Geopolymeric Gels From alkali-Feldspars. Colloids and Surfaces A: Physicochemical Engineer Aspects, v. 216, p. 27-44, 2003. 
YOST, J. R. et al. Structural Behaviour of Alkali Activated Fly Ash concrete: part 1: mixture design, material properties and sample fabrication. Materials and Structures, v. 46, p. 435-447, 2013.

\section{Agradecimentos}

Agradecimento aos colegas do laboratório Nanotec Lucas Onghero e Ronaldo Pilar; do Laboratório LMCC, Roque e Luiz. Ao CNPq, pelo auxílio financeiro na forma de bolsa de mestrado.

\section{Carolina Noda Livi}

Centro Tecnológico, Departamento de Engenharia Civil | Universidade Federal de Santa Catarina | Rua João Pio Duarte Silva, s/n, Córrego Grande | Campus Trindade, Trindade | Florianópolis - SC - Brasil | CEP 88010-970 | Tel.: (48) 3721-9370 | E-mail: carolinanlivi@gmail.com

\section{Wellington Longuini Repette}

Centro Tecnológico, Departamento de Engenharia Civil | Universidade Federal de Santa Catarina | E-mail: wellington.repette@gmail.com

\section{Revista Ambiente Construído}

Associação Nacional de Tecnologia do Ambiente Construído

Av. Osvaldo Aranha, $99-3^{\circ}$ andar, Centro

Porto Alegre - RS - Brasil

$$
\text { CEP } 90035-190
$$

Telefone: +55 (51) 3308-4084

Fax: +55 (51) 3308-4054

www.seer.ufrgs.br/ambienteconstruido

E-mail: ambienteconstruido@ufrgs.br 